\title{
Risk Minimization and Optimal Derivative Design in a Principal Agent Game*
}

\author{
Ulrich Horst \\ Department of Mathematics \\ Humboldt University Berlin \\ Unter den Linden 6 \\ 10099 Berlin \\ horst@math.hu-berlin.de
}

\author{
Santiago Moreno-Bromberg \\ Department of Mathematics \\ University of British Columbia \\ 1984 Mathematics Road \\ Vancouver, BC, V6T $1 \mathrm{Z2}$ \\ smoreno@math.ubc.ca
}

October 29, 2018

\begin{abstract}
We consider the problem of Adverse Selection and optimal derivative design within a Principal-Agent framework. The principal's income is exposed to non-hedgeable risk factors arising, for instance, from weather or climate phenomena. She evaluates her risk using a coherent and law invariant risk measure and tries minimize her exposure by selling derivative securities on her income to individual agents. The agents have mean-variance preferences with heterogeneous risk aversion coefficients. An agent's degree of risk aversion is private information and hidden to the principal who only knows the overall distribution. We show that the principal's risk minimization problem has a solution and illustrate the effects of risk transfer on her income by means of two specific examples. Our model extends earlier work of Barrieu and El Karoui (2005) and Carlier, Ekeland and Touzi (2007).
\end{abstract}

\section{Preliminary - Comments Welcome}

AMS classification: 60G35, 60H20, 91B16, 91B70.

Keywords: Optimal derivative design, structured securities, adverse selection, risk transfer.

\footnotetext{
*We thank Guillaume Carlier, Pierre-Andre Chiappori, Ivar Ekeland, Andreas Putz and seminar participants at various institutions for valuable comments and suggestions. Financial support through an NSERC individual discovery grant is gratefully acknowledged.
} 


\section{Introduction}

In recent years there has been an increasing interest in derivative securities at the interface of finance and insurance. Structured products such as risk bonds, asset-backed securities and weather derivatives are end-products of a process known as securitization that transforms non-tradable risk factors into tradable financial assets. Developed in the U.S. mortgage markets, the idea of pooling and underwriting risk that cannot be hedged through investments in the capital markets alone has long become a key factor driving the convergence of insurance and financial markets.

Structured products are often written on non-tradable underlyings, tailored to the issuers specific needs and traded "over the counter". Insurance companies, for instance, routinely sell weather derivatives or risk bonds to customers that cannot go to the capital markets directly and/or seek financial securities with low correlation with stock indices as additions to diversified portfolios. The market for such claims is generally incomplete and illiquid. As a result, many of the standard paradigms of traditional derivative pricing theory, including replication arguments do not apply to structured products. In an illiquid market framework, preference-based valuation principles that take into account characteristics and endowment of trading partners may be more appropriate for designing, pricing and hedging contingent claims. Such valuation principles have become a major topic of current research in economics and financial mathematics. They include rules of Pareto optimal risk allocation ([11], [16]), market completion and dynamic equilibrium pricing ([14], [15]) and, in particular, utility indifference arguments ([2], [3], [5], [6], [9], ...). The latter assumes a high degree of market asymmetry. For indifference valuation to be a pricing rather than valuation principle, the demand for a financial security must come from identical agents with known preferences and negligible market impact while the supply must come from a single principal. When the demand comes from heterogeneous individuals with hidden characteristics, indifference arguments do not always yield an appropriate pricing scheme.

In this paper we move away from the assumption of investor homogeneity and allow for heterogeneous agents. We consider a single principal with a random endowment whose goal is to lay off some of her risk with heterogeneous agents by designing and selling derivative securities on her income. The agents have mean variance preferences. An agent's degree of risk aversion is private information and hidden to the principal. The principal only knows the distribution of risk aversion coefficients which puts her at an informational disadvantage. If all the agents were homogeneous, the principal, when offering a structured product to a single agent, could (perhaps) extract the indifference (maximum) price from each trading partner. In the presence of agent heterogeneity this is no longer possible, either because the agents would hide their characteristics from the principal or prefer another asset offered by the principal but designed and priced for another customer.

The problem of optimal derivative design in a Principal-Agent framework with informed agents and an uninformed principal has first been addressed in a recent paper by of Carlier, Ekeland 
and Touzi [7]. With the agents being the informed party, theirs is a screening model. The literature on screening within the Adverse Selection framework can be traced back to Mussa and Rosen [18, where both the principal's allocation rule and the agents' types are one-dimensional. Armstrong [1] relaxes the hypothesis of agents being characterized by a single parameter. He shows that, unlike the one-dimensional case, "bunching" of the first type is robust when the types of the agents are multi-dimensional. In their seminal paper, Rochet and Choné [19] further extend this analysis. They provide a characterization of the contracts, determined by the (nonlinear) pricing schedule, that maximize the principal's utility under the constraints imposed by the asymmetry of information in the models. Building on their work, Carlier, Ekeland and Touzi [7] study a Principal-Agent model of optimal derivative design where the agents' preferences are of mean-variance type and their multi-dimensional types characterize their risk aversions and initial endowments. They assume that there is a direct cost to the principal when she designs a contract for an agent, and that the principal's aim is to maximize profits.

We start from a similar set-up, but substitute the idea that providing products carries a cost for the idea that traded contracts expose the principal to additional risk - as measured by a convex risk measure - in exchange for a known revenue. This may be viewed as a partial extension of the work by Barrieu and El Karoui ([2], [3] ) to an incomplete information framework.

The principal's aim is to minimize her risk exposure by trading with the agents subject to the standard incentive compatibility and individual rationality conditions on the agents' choices. In order to prove that the principal's risk minimization problem has a solution we first follow the seminal idea of Rochet and Choné [19] and characterize incentive compatible catalogues in terms of $U$-convex functions. When the impact of a single trade on the principal's revenues is linear as in Carlier, Ekeland and Touzi [7], the link between incentive compatibility and $U$-convexity is key to establish the existence of an optimal solution. In our model the impact is non-linear as a single trade has a non-linear impact on the principal's risk assessment. Due to this non-linearity we face a non-standard variational problem where the objective cannot be written as the integral of a given Lagrangian. Instead, our problem can be decomposed into a standard variational part representing the aggregate income of the principal, plus the minimization of the principal's risk evaluation, which depends on the aggregate of the derivatives traded. We state sufficient conditions that guarantee that the principal's optimization problem has a solution and illustrate the effect of risk transfer on her exposure by means of two specific examples.

The remainder of this paper is organized as follows. In Section 2 we formulate our PrincipalAgent model and state the main result. The proof is given in Section 3. In Section 4 we illustrate the effects of risk transfer on the principal's position by two examples. In the first we consider a situation where the principal restricts itself to type-dependent multiples of some benchmark claim. This case can be solved in closed form by means of a standard variational problem. The second example considers put options with type-dependent strikes. In both cases we assume that 
the principal's risk measure is Average Value at Risk. As a consequence the risk minimization problem can be stated in terms of a min-max problem; we provide an efficient numerical scheme for approximating the optimal solution. The code is given in an appendix.

\section{The Microeconomic Setup}

We consider an economy with a single principal whose income $W$ is exposed to non-hedgeable risk factors rising from, e.g., climate or weather phenomena. The random variable $W$ is defined on a standard, non-atomic, probability space $(\Omega, \mathcal{F}, \mathbb{P})$ and it is square integrable:

$$
W \in L^{2}(\Omega, \mathcal{F}, \mathbb{P}) .
$$

The principal's goal is to lay off parts of her risk with individual agents. The agents have heterogenous mean-variance preferences 1 and are indexed by their coefficients of risk aversion $\theta \in \Theta$. Given a contingent claim $Y \in L^{2}(\Omega, \mathcal{F}, \mathbb{P})$ an agent of type $\theta$ enjoys the utility

$$
U(\theta, Y)=\mathbb{E}[Y]-\theta \operatorname{Var}[Y]
$$

Types are private information. The principal knows the distribution $\mu$ of types but not the realizations of the random variables $\theta$. We assume that the agents are risk averse and that the risk aversion coefficients are bounded away from zero. More precisely,

$$
\Theta=[a, 1] \quad \text { for some } a>0 \text {. }
$$

The principal offers a derivative security $X(\theta)$ written on her random income for any type $\theta$. The set of all such securities is denoted by

$$
\mathcal{X}:=\left\{X=\{X(\theta)\}_{\theta \in \Theta} \mid X \in L^{2}(\Omega \times \Theta, \mathbb{P} \otimes \mu), X \text { is } \sigma(W) \times \mathcal{B}(\Theta) \text { measurable }\right\} .
$$

We refer to a list of securities $\{X(\theta)\}$ as a contract. A catalogue is a contract along with prices $\pi(\theta)$ for every available derivative $X(\theta)$. For a given catalogue $(X, \pi)$ the optimal net utility of the agent of type $\theta$ is given by

$$
v(\theta)=\sup _{\theta^{\prime} \in \Theta}\left\{U\left(\theta, X\left(\theta^{\prime}\right)\right)-\pi\left(\theta^{\prime}\right)\right\}
$$

Remark 2.1 No assumption will be made on the sign of $\pi(\theta)$; our model contemplates both the case where the principal takes additional risk in exchange of financial compensation and the one where she pays the agents to take part of her risk.

\footnotetext{
${ }^{1}$ Our analysis carries over to preferences of mean-variance type with random initial endowment as in [7]; the assumption of simple mean-variance preferences is made for notational convenience.
} 
A catalogue $(X, \pi)$ will be called incentive compatible (IC) if the agent's interests are best served by revealing her type. This means that her optimal utility is achieved by the security $X(\theta)$ :

$$
U(\theta, X(\theta))-\pi(\theta) \geq U\left(\theta, X\left(\theta^{\prime}\right)\right)-\pi\left(\theta^{\prime}\right) \text { for all } \theta, \theta^{\prime} \in \Theta
$$

We assume that each agent has some outside option ("no trade") that yields a utility of zero. A catalogue is thus called individually rational (IR) if it yields at least the reservation utility for all agents, i.e., if

$$
U(\theta, X(\theta))-\pi(\theta) \geq 0 \text { for all } \theta \in \Theta .
$$

Remark 2.2 By offering only incentive compatible contracts, the principal forces the agents to reveal their type. Offering contracts where the IR constraint is binding allows the principal to exclude undesirable agents from participating in the market. It can be shown that under certain conditions, the interests of the Principal are better served by keeping agents of "lower types" to their reservation utility; Rochet and Choné [19] have shown that in higher dimensions this is always the case.

If the principal issues the catalogue $(X, \pi)$, she receives a cash amount of $\int_{\Theta} \pi(\theta) d \mu(\theta)$ and is subject to the additional liability $\int_{\Theta} X(\theta) \mu(d \theta)$. She evaluates the risk associated with her overall position

$$
W+\int_{\Theta}(\pi(\theta)-X(\theta)) d \mu(\theta)
$$

via a coherent and law-invariant risk measure $\varrho: L^{2}(\Omega, \mathcal{F}, \mathbb{P}) \rightarrow \mathbb{R} \cup\{\infty\}$ that has the Fatou property. It turns out that such risk measures can be represented as robust mixtures of Average Value at Risk 2 The principal's risk associated with the catalogue $(X, \pi)$ is given by

$$
\varrho\left(W+\int_{\Theta}(\pi(\theta)-X(\theta)) d \mu(\theta)\right) .
$$

Her goal is to devise contracts $(X, \pi)$ that minimize (6) subject to the incentive compatibility and individual rationality condition:

$$
\inf \left\{\varrho\left(W+\int_{\Theta}(\pi(\theta)-X(\theta)) d \mu(\theta)\right) \mid X \in \mathcal{X}, X \text { is } \mathbf{I C} \text { and } \mathbf{I R}\right\} .
$$

We are now ready to state the main result of this paper. The proof requires some preparation and will be carried out in the following section.

Theorem 2.3 If $\varrho$ is a coherent and law invariant risk measure on $L^{2}(\mathbb{P})$ and if $\varrho$ has the Fatou property, then the principal's optimization problem has a solution.

For notational convenient we establish our main result for the spacial case $d \mu(\theta)=d \theta$. The general case follows from straight forward modifications.

\footnotetext{
${ }^{2}$ We review properties of coherent risk measures on $L^{p}$ spaces in the appendix and refer to the textbook by Föllmer and Schied [12] and the paper of Jouini, Schachermayer and Touzi [17] for detailed discussion of law invariant risk measures.
} 


\section{Proof of the Main Theorem}

Let $(X, \pi)$ be a catalogue. In order to prove our main result it will be convenient to assume that the principal offers any square integrable contingent claim and to view the agents' optimization problem as optimization problems over the set $L^{2}(\mathbb{P})$. This can be achieved by identifying the price list $\{\pi(\theta)\}$ with the pricing scheme

$$
\pi: L^{2}(\mathbb{P}) \rightarrow \mathbb{R}
$$

that assigns the value $\pi(\theta)$ to an available claim $X(\theta)$ and the value $\mathbb{E}[Y]$ to any other claim $Y \in L^{2}$. In terms of this pricing scheme the value function $v$ defined in (3) satisfies

$$
v(\theta)=\sup _{Y \in L^{2}(\mathbb{P})}\{U(\theta, Y)-\pi(Y)\} .
$$

for any individually rational catalogue. For the remainder of this section we shall work with the value function of the (8). It is $U$-convex in the sense of the following definition; it actually turns out to be convex and non-increasing as we shall prove in Proposition 3.2 below.

Definition 3.1 Let two spaces $A$ and $B$ and a function $U: A \times B \rightarrow \mathbb{R}$ be given.

(i) The function $f: A \rightarrow \mathbb{R}$ is called $U$-convex if there exists a function $p: B \rightarrow \mathbb{R}$ such that

$$
f(a)=\sup _{b \in B}\{U(a, b)-p(b)\}
$$

(ii) For a given function $p: B \rightarrow \mathbb{R}$ the $U$-conjugate $p^{U}(a)$ of $p$ is defined by

$$
p^{U}(a)=\sup _{b \in B}\{U(a, b)-p(b)\} .
$$

(iii) The U-subdifferential of $p$ at $b$ is given by the set

$$
\partial_{U} p(b):=\left\{a \in A \mid p^{U}(a)=U(a, b)-p(b)\right\} .
$$

(iv) If $a \in \partial_{U} p(b)$, then a is called a U-subgradient of $p(b)$.

Our goal is to identify the class of IC and IR catalogues with a class of convex and nonincreasing functions on the type space. To this end, we first recall the link between incentive compatible contracts and $U$-convex functions from Rochet and Choné [19] and Carlier, Ekeland and Touzi [7].

Proposition 3.2 ([19], [7]) If a catalogue $(X, \pi)$ is incentive compatible, then the function $v$ defined by (3) is proper and U-convex and $X(\theta) \in \partial_{U} v(\theta)$. Conversely, any proper, U-convex function induces an incentive compatible catalogue. 
Proof. Incentive compatibility of a catalogue $(X, \pi)$ means that

$$
U(\theta, X(\theta))-\pi(\theta) \geq U\left(\theta, X\left(\theta^{\prime}\right)\right)-\pi\left(\theta^{\prime}\right) \text { for all } \theta, \theta^{\prime} \in \Theta
$$

so $v(\theta)=U(\theta, X(\theta))-\pi(\theta)$ is U-convex and $X(\theta) \in \partial_{U} v(\theta)$. Conversely, for a proper, U-convex function $v$ and $X(\theta) \in \partial_{U} v(\theta)$ let

$$
\pi(\theta):=U(\theta, X(\theta))-v(\theta)
$$

By the definition of the U-subdifferential, the catalogue $(X, \pi)$ is incentive compatible.

The following lemma is key. It shows that the $U$-convex function $v$ is convex and non-increasing and that any convex and non-increasing function is $U$-convex, i.e., it allows a representation of the form (8). This allows us to rephrase the principal's problem as an optimization problem over a compact set of convex functions.

Lemma 3.3 (i) Suppose that the value function $v$ as defined by (8) is proper. Then $v$ is convex and non-increasing. Any optimal claim $X^{*}(\theta)$ is a $U$-subgradient of $v(\theta)$ and almost surely

$$
-\operatorname{Var}\left[X^{*}(\theta)\right]=v^{\prime}(\theta) \text {. }
$$

(ii) If $\bar{v}: \Theta \rightarrow \mathbb{R}_{+}$is proper, convex and non-increasing, then $\bar{v}$ is $U$-convex, i.e., there exists a map $\bar{\pi}: L^{2}(\mathbb{P}) \rightarrow \mathbb{R}$ such that

$$
\bar{v}(\theta)=\sup _{Y \in L^{2}(\mathbb{P})}\{U(\theta, Y)-\bar{\pi}(Y)\} .
$$

Furthermore, any optimal claim $\bar{X}(\theta)$ belongs to the $U$-subdifferential of $\bar{v}(\theta)$ and satisfies

$$
-\operatorname{Var}[\bar{X}(\theta)]=\bar{v}^{\prime}(\theta) \text {. }
$$

ProOF.

(i) Let $v$ be a proper, $U$-convex function. Its $U$-conjugate is:

$$
\begin{aligned}
v^{U}(Y) & =\sup _{\theta \in \Theta}\{\mathbb{E}[Y]-\theta \operatorname{Var}[Y]-v(\theta)\} \\
& =\mathbb{E}[Y]+\sup _{\theta \in \Theta}\{\theta(-\operatorname{Var}[Y])-v(\theta)\} \\
& =\mathbb{E}[Y]+v^{*}(-\operatorname{Var}[Y]),
\end{aligned}
$$

where $v^{*}$ denotes the convex conjugate of $v$. As a $U$-convex function, the map $v$ is characterized by the fact that $v=\left(v^{U}\right)^{U}$. Thus

$$
\begin{aligned}
v(\theta) & =\left(v^{U}\right)^{U}(\theta) \\
& =\sup _{Y \in L^{2}(\mathbb{P})}\left\{U(\theta, Y)-\mathbb{E}[Y]-v^{*}(-\operatorname{Var}[Y])\right\} \\
& =\sup _{Y \in L^{2}(\mathbb{P})}\left\{\mathbb{E}[Y]-\theta \operatorname{Var}[Y]-\mathbb{E}[Y]-v^{*}(-\operatorname{Var}[Y])\right\} \\
& =\sup _{y \leq 0}\left\{\theta \cdot y-v^{*}(y)\right\}
\end{aligned}
$$


where the last equality uses the fact that the agents' consumption set contains claims of any variance. We deduce from the preceding representation that $v$ is non-increasing. Furthermore $v=\left(v^{*}\right)^{*}$ so $v$ is convex. To characterize $\partial_{U} v(\theta)$ we proceed as follows:

$$
\begin{aligned}
\partial_{U} v(\theta) & =\left\{Y \in L^{2} \mid v(\theta)=U(\theta, X)-v^{U}(Y)\right\} \\
& =\left\{Y \in L^{2} \mid v(\theta)=\mathbb{E}[Y]-\theta \operatorname{Var}[Y]-v^{U}(Y)\right\} \\
& =\left\{Y \in L^{2} \mid v(\theta)=\mathbb{E}[Y]-\theta \operatorname{Var}[Y]-\mathbb{E}[Y]-v^{*}(-\operatorname{Var}[Y])\right\} \\
& =\left\{Y \in L^{2} \mid v(\theta)=\theta(-\operatorname{Var}[Y])-v^{*}(-\operatorname{Var}[Y])\right\} \\
& =\left\{Y \in L^{2} \mid-\operatorname{Var}[Y] \in \partial v(\theta)\right\}
\end{aligned}
$$

The convexity of $v$ implies it is a.e. differentiable so we may write

$$
\left.\partial_{U} v(\theta):=\left\{Y \in L^{2} \mid v^{\prime}(\theta)=-\operatorname{Var}[Y]\right)\right\} .
$$

(ii) Let us now consider a proper, non-negative, convex and non-increasing function $\bar{v}: \Theta \rightarrow \mathbb{R}$. There exists a map $f: \mathbb{R} \rightarrow \mathbb{R}$ such that

$$
\bar{v}(\theta)=\sup _{y \leq 0}\{\theta \cdot y-f(y)\} .
$$

Since $\bar{v}$ is non-increasing there exists a random variable $Y(\theta) \in L^{2}(\mathbb{P})$ such that $-\operatorname{Var}[Y(\theta)] \in$ $\partial \bar{v}(\theta)$ and the definition of the subgradient yields

$$
\bar{v}(\theta)=\sup _{Y \in L^{2}}\{\theta(-\operatorname{Var}[Y])-f(-\operatorname{Var}[Y])\} .
$$

With the pricing scheme on $L^{2}(\mathbb{P})$ defined by

$$
\bar{\pi}(Y):=-\mathbb{E}[Y]-f(-\operatorname{Var}[Y])
$$

this yields

$$
\bar{v}(\theta)=\sup _{Y \in L^{2}}\{U(\theta, Y)-\bar{\pi}(Y)\} .
$$

The characterization of the subdifferential follows by analogy to part (i).

The preceding lemma along with Proposition 3.2 shows that any convex, non-negative and non-increasing function $v$ on $\Theta$ induces an incentive compatible catalogue $(X, \pi)$ via

$$
X(\theta) \in \partial_{U} v(\theta) \quad \text { and } \quad \pi(\theta)=U(\theta, X(\theta))-v(\theta) .
$$

Here we may with no loss of generality assume that $\mathbb{E}[X(\theta)]=0$. In terms of the principal's choice of $v$ her income is given by

$$
I(v)=\int_{\Theta}\left(\theta v^{\prime}(\theta)-v(\theta)\right) d \theta .
$$


Since $v \geq 0$ is decreasing and non-negative the principal will only consider functions that satisfy the normalization constraint

$$
v(1)=0
$$

We denote the class of all convex, non-increasing and non-negative real-valued functions on $\Theta$ that satisfy the preceding condition by $\mathcal{C}$ :

$$
\mathcal{C}=\{v: \Theta \rightarrow \mathbb{R} \mid v \text { is convex, non-increasing, non-negative and } v(1)=0 .\}
$$

Conversely, we can associate with any $\mathbf{I C}$ and $\mathbf{I R}$ catalogue $(X, \pi)$ a non-negative $U$-convex function of the form (8) where the contract satisfies the variance constraint - $\operatorname{Var}[X(\theta)]=v^{\prime}(\theta)$. In view of the preceding lemma this function is convex and non-increasing so after normalization we may assume that $v$ belongs to the class $\mathcal{C}$. We therefore have the following alternative formulation of the principal's problem.

Theorem 3.4 The principal's optimization problem allows the following alternative formulation:

$$
\inf \left\{\varrho\left(W-\int_{\Theta} X(\theta) d \theta\right)-I(v) \mid v \in \mathcal{C}, \mathbb{E}[X(\theta)]=0,-\operatorname{Var}[X(\theta)]=v^{\prime}(\theta)\right\} .
$$

In terms of our alternative formulation we can now prove a preliminary result. It states that a principal with no initial endowment will not issue any contracts.

Lemma 3.5 If the principal has no initial endowment, i.e., if $W=0$, then $(v, X)=(0,0)$ solves her optimization problem.

Proof. Since $\varrho$ is a coherent, law invariant risk measure on $L^{2}(\mathbb{P})$ that has the Fatou property it satisfies

$$
\varrho(Y) \geq-\mathbb{E}[Y] \text { for all } Y \in L^{2}(\mathbb{P}) .
$$

For a given function $v \in \mathcal{C}$ the normalization constraint $\mathbb{E}[X(\theta)]=0$ therefore yields

$$
\varrho\left(-\int_{\Theta} X(\theta) d \theta\right)-I(v) \geq \mathbb{E}\left[\int_{\Theta} X(\theta) d \theta\right]-I(v)=-I(v) .
$$

Since $v$ is non-negative and non-increasing $-I(v) \geq 0$. Taking the infimum in the preceding inequality shows that $v \equiv 0$ and hence $X(\theta) \equiv 0$ is an optimal solution.

\subsection{Minimizing the risk for a given function $v$}

In the general case we approach the principal's problem in two steps. We start by fixing a function $v$ from the class $\mathcal{C}$ and minimize the associated risk

$$
\varrho\left(W-\int_{\Theta} X(\theta) d \theta\right)
$$


subject to the moment conditions $\mathbb{E}[X(\theta)]=0$ and $-\operatorname{Var}[X(\theta)]=v^{\prime}(\theta)$. To this end, we shall first prove the existence of optimal contracts $X_{v}$ for a relaxed optimization where the variance constraint is replaced by the weaker condition

$$
\operatorname{Var}[X(\theta)] \leq-v^{\prime}(\theta)
$$

In a subsequent step we show that based on $X_{v}$ the principal can transfer risk exposures among the agents in such a way that (i) the aggregate risk remains unaltered; (ii) the variance constraint becomes binding. We assume with no loss of generality that $v$ does not have a jump at $\theta=a$.

\subsubsection{The relaxed optimization problem}

For a given $v \in \mathcal{C}$ let us consider the convex set of derivative securities

$$
\mathcal{X}_{v}:=\left\{X \in \mathcal{X} \mid E[X(\theta)]=0, \operatorname{Var}[X(\theta)] \leq-v^{\prime}(\theta) \mu-\text { a.e. }\right\} .
$$

Lemma 3.6 (i) All functions $v \in \mathcal{C}$ that are acceptable for the principal are uniformly bounded.

(ii) Under the conditions of (i) the set $\mathcal{X}_{v}$ is closed and bounded in $L^{2}(\mathbb{P} \otimes \mu)$. More precisely,

$$
\|X\|_{2}^{2} \leq v(a) \quad \text { for all } \quad X \in \mathcal{X}_{v} .
$$

ProOF.

(i) If $v$ is acceptable for the principal, then any $X \in \mathcal{X}_{v}$ satisfies

$$
\varrho\left(W-\int_{\Theta} X(\theta) d \theta\right)-I(v) \leq \varrho(W) .
$$

From (9) and that fact that $\mathbb{E}[X(\theta)]=0$ we deduce that

$$
-\mathbb{E}[W]-I(v) \leq \varrho\left(W-\int_{\Theta} X(\theta) d \theta\right)-I(v) \leq \varrho(W)
$$

so

$$
-I(v) \leq \mathbb{E}[W]+\varrho(W)=: K .
$$

Integrating by parts twice and using that $v$ is non-increasing and $v(1)=0$ we see that

$$
K \geq-I(v)=a v(a)+2 \int_{a}^{1} v(\theta) d \theta \geq a v(a) .
$$

This proves the assertion because $a>0$.

(ii) For $X \in \mathcal{X}_{v}$ we deduce from the normalization constraint $v(1)=0$ that

$$
\|X\|_{2}^{2}=\iint X^{2}(\theta, \omega) d \mathbb{P} d \theta \leq-\int v^{\prime}(\theta) d \theta \leq v(a)
$$

so the assertion follows from part (i). 
Since $\varrho$ is a convex risk measure on $L^{2}$ and because the set $X_{v}$ of contingent claims is convex, closed and bounded in $L^{2}$ a general result from the theory of convex optimization yields the following proposition.

Proposition 3.7 If the function $v$ is acceptable for the principal, then there exists a contract $\left\{X_{v}(\theta)\right\}$ such that

$$
\inf _{X \in \mathcal{X}_{v}} \varrho\left(W-\int_{\Theta} X(\theta) d \theta\right)=\varrho\left(W-\int_{\Theta} X_{v}(\theta) d \theta\right)
$$

The contract $X_{v}$ along with the pricing scheme associated with $v$ does not yield an incentive compatible catalogue unless the variance constraints happen to be binding. However, as we are now going to show, based on $X_{v}$ the principal can find a redistribution of risk among the agents such that the resulting contract satisfies our IC condition.

\subsubsection{Redistributing risk exposures among agents}

Let

$$
\partial \mathcal{X}_{v}=\left\{X \in \mathcal{X}_{v} \mid E[X(\theta)]=0, \operatorname{Var}[X(\theta)]=-v^{\prime}(\theta), \mu-\text { a.e. }\right\}
$$

be the set of all contracts from the class $\mathcal{X}_{v}$ where the variance constraint is binding. Clearly,

$$
\varrho\left(W-\int_{\Theta} X_{v}(\theta) d \theta\right) \leq \inf _{X \in \partial \mathcal{X}} \varrho\left(W-\int_{\Theta} X(\theta) d \theta\right)
$$

Let us then introduce the set of types

$$
\Theta_{v}:=\left\{\theta \in \Theta \mid \operatorname{Var}\left[X_{v}(\theta)\right]<-v^{\prime}(\theta)\right\},
$$

for whom the variance constraint is not binding. If $\mu\left(\Theta_{v}\right)=0$, then $X_{v}$ yields an incentive compatible contract. Otherwise, we consider a random variable $\tilde{Y} \in \mathcal{X}_{v}$, fix some type $\bar{\theta} \in \Theta$ and define

$$
Y:=\frac{\tilde{Y}(\bar{\theta})}{\sqrt{\operatorname{Var}[\tilde{Y}(\bar{\theta})]}} .
$$

We may with no loss of generality assume that $Y$ is well defined for otherwise the status quo is optimal for the principal and her risk minimization problem is void. The purpose of introducing $Y$ is to offer a set of structured products $Z_{v}$ based on $X_{v}$, such that $Z_{v}$ together with the pricing scheme associated with $v$ yields an incentive compatible catalogue. To this end, we choose constants $\tilde{\alpha}(\theta)$ for $\theta \in \Theta_{v}$ such that

$$
\operatorname{Var}\left[X_{v}(\theta)+\tilde{\alpha}(\theta) Y\right]=-v^{\prime}(\theta)
$$


This equation holds for

$$
\tilde{\alpha}_{ \pm}(\theta)=-\operatorname{Cov}\left[X_{v}(\theta), Y\right] \pm \sqrt{\operatorname{Cov}^{2}\left[X_{v}(\theta), Y\right]-v^{\prime}(\theta)-\operatorname{Var}\left[X_{v}(\theta)\right]} .
$$

For a type $\theta \in \Theta_{v}$ the variance constraint is not binding. Hence $-v^{\prime}(\theta)-\operatorname{Var}\left[X_{v}(\theta)\right]>0$ so that $\alpha_{+}(\theta)>0$ and $\alpha_{-}(\theta)<0$. An application of Jensen's inequality together with the fact that $\left\|X_{v}\right\|_{2}$ is bounded shows that $\alpha_{ \pm}$are $\mu$-integrable functions. Thus there exists a threshold type $\theta^{*} \in \Theta$ such that

$$
\int_{\Theta_{v} \cap\left(a, \theta^{*}\right]} \alpha^{+}(\theta) d \theta+\int_{\Theta_{v} \cap\left(\theta^{*}, 1\right]} \alpha^{-}(\theta) d \theta=0 .
$$

In terms of $\theta^{*}$ let us now define a function

$$
\alpha(\theta):= \begin{cases}\tilde{\alpha}^{+}(\theta), & \text { if } \theta \leq \theta^{*} \\ \tilde{\alpha}^{-}(\theta), & \text { if } \theta>\theta^{*}\end{cases}
$$

and a contract

$$
Z_{v}:=X_{v}+\alpha Y \in \partial \mathcal{X}_{v}
$$

Since $\int \alpha d \theta=0$ the aggregate risks associated with $X_{v}$ and $Z_{v}$ are equal. As a result, the contract $Z_{v}$ solves the risk minimization problem

$$
\inf _{X \in \partial \mathcal{X}_{v}} \varrho\left(W+\int_{\Theta} X(\theta) \mu(d \theta)\right) .
$$

Remark 3.8 In Section 4 we shall consider a situation where the principal restricts itself to a class of contracts for which the random variable $X_{v}$ can be expressed in terms of the function $v$. In general such a representation will not be possible since $v$ only imposes a restriction on the contracts' second moments.

\subsection{Minimizing the overall risk}

In order to finish the proof of our main result it remains to show that the minimization problem

$$
\inf _{v \in \mathcal{C}}\left\{\varrho\left(W-\int_{\Theta} Z_{v}(\theta) \mu(d \theta)\right)-I(v)\right\}
$$

has a solution and the infimum is obtained. To this end, we consider a minimizing sequence $\left\{v_{n}\right\} \subset \mathcal{C}$. The functions in $\mathcal{C}$ are locally Lipschitz continuous because they are convex. In fact they are uniformly locally Lipschitz: by Lemma 3.6 (i) the functions $v \in \mathcal{C}$ are uniformly bounded and non-increasing so all the elements of $\partial v(\theta)$ are uniformly bounded on compact sets of types. As a result, $\left\{v_{n}\right\}$ is a sequence of uniformly bounded and uniformly equicontinuous functions when restricted to compact subsets of $\Theta$. Thus there exists a function $\bar{v} \in \mathcal{C}$ such that, passing to a subsequence if necessary,

$$
\lim _{n \rightarrow \infty} v_{n}=\bar{v} \quad \text { uniformly on compact sets. }
$$


A standard $3 \epsilon$-argument shows that the convergence properties of the sequence $\left\{v_{n}\right\}$ carry over to the derivatives so that

$$
\lim _{n \rightarrow \infty} v_{n}^{\prime}=\bar{v}^{\prime} \quad \text { almost surely uniformly on compact sets. }
$$

Since $-\theta v_{n}^{\prime}(\theta)+v_{n}(\theta) \geq 0$ it follows from Fatou's lemma that $-I(\bar{v}) \leq \liminf _{n \rightarrow \infty}-I\left(v_{n}\right)$ so

$$
\begin{aligned}
& \liminf _{n \rightarrow \infty}\left\{\varrho\left(W-\int_{\Theta} Z_{v_{n}}(\theta) \mu(d \theta)\right)-I\left(v_{n}\right)\right\} \\
\geq & \liminf _{n \rightarrow \infty} \varrho\left(W-\int_{\Theta} Z_{v_{n}}(\theta) \mu(d \theta)\right)+\liminf _{n \rightarrow \infty}-I\left(v_{n}\right) \\
\geq & \liminf _{n \rightarrow \infty} \varrho\left(W-\int_{\Theta} Z_{v_{n}}(\theta) \mu(d \theta)\right)-I(\bar{v})
\end{aligned}
$$

and it remains to analyze the associated risk process. For this, we first observe that for $Z_{v_{n}} \in \partial X_{v_{n}}$ Fubini's theorem yields

$$
\left\|Z_{v_{n}}\right\|_{2}^{2}=\iint Z_{v_{n}}^{2} d \mathbb{P} d \theta=-\int v_{n}^{\prime}(\theta) d \theta=v_{n}(a) .
$$

Since all the functions in $\mathcal{C}$ are uniformly bounded, we see that the contracts $Z_{v_{n}}$ are contained in an $L^{2}$ bounded, convex set. Hence there exists a square integrable random variable $Z$ such that, after passing to a subsequence if necessary,

$$
w-\lim _{n \rightarrow \infty} Z_{n}=Z
$$

Let $Z_{\bar{v}} \in X_{\bar{v}}$. Convergence of the functions $v_{n}$ implies $\left\|Z_{v_{n}}\right\|_{2} \rightarrow\left\|Z_{\bar{v}}\right\|_{2}$. Thus (15) yields $\|Z\|_{2}=\left\|Z_{\bar{v}}\right\|_{2}$ along with convergence of aggregate risks:

$$
\|Z\|_{2}=\left\|Z_{\bar{v}}\right\|_{2} \quad \text { and } \quad \int_{\Theta} Z_{n}(\theta, \omega) d \theta \rightarrow \int_{\Theta} Z(\theta, \omega) d \theta \quad \text { weakly in } L^{2}(\mathbb{P}) .
$$

By Corollary I.2.2 in Ekeland and Témam (1976) [10], a lower semi-continuous convex function $f: X \rightarrow \mathbb{R}$ remains ,so with respect to the weak topology $\sigma\left(X, X^{*}\right)$, the Fatou property of the risk measure $\varrho$ guarantees that

$$
\begin{aligned}
\varrho\left(W-\int_{\Theta} Z_{\bar{v}}(\theta) \mu(d \theta)\right) & \leq \varrho\left(W-\int_{\Theta} Z(\theta) \mu(d \theta)\right) \\
& \leq \liminf _{n \rightarrow \infty} \varrho\left(W-\int_{\Theta} Z(\theta) \mu(d \theta)\right) .
\end{aligned}
$$

We conclude that $\left(Z_{\bar{v}}, \bar{v}\right)$ solves the Principal's problem. 


\section{Examples}

Our main theorem states that the principal's risk minimization problem has a solution. The solution can be characterized in terms of a convex function that specifies the agents' net utility. Our existence result is based on a min-max optimization scheme whose complexity renders a rather involved numerical analysis. In this section we consider some examples where the principal's choice of contracts is restricted to class of numerically more amenable securities. The first example studies a situation where the principal offers type-dependent multiplies of some benchmark claim. In this case the principal's problem can be reduced to a constraint variational problem that can be solved in closed form. A second example comprises put options with type dependent strikes. Here we provide a numerical algorithm for approximating the optimal solution.

\subsection{A single benchmark claim}

In this section we study a model where the principal sells a type-dependent multiple of a benchmark claim $f(W) \geq 0$ to the agents. More precisely, the principal offers contracts of the form

$$
X(\theta)=\alpha(\theta) f(W) .
$$

In order to simplify the notation we shall assume that the T-bond's variance is normalized:

$$
\operatorname{Var}[f(W)]=1
$$

\subsubsection{The optimization problems}

Let $(X, \pi)$ be a catalogue where the contract $X$ is of the form (16). By analogy to the general case it will be convenient to view the agents' optimization problem as an optimization problem of the set of claims $\{\gamma f(W) \mid \gamma \in \mathbb{R}\}$ so the function $\alpha: \Theta \rightarrow \mathbb{R}$ solves

$$
\sup _{\gamma \in \mathbb{R}}\{U(\theta, \gamma f(W))-\pi(\theta)\} .
$$

In view of the variance constraint on the agents' claims the principal's problem can be written as

$$
\inf \{\varrho(W-C(v) f(W))-I(v) \mid v \in \mathcal{C}\} \quad \text { where } \quad C(v)=\int_{\Theta} \sqrt{-v^{\prime}(\theta)} d \theta .
$$

Note that $E[f(W)]>0$, so the term $E[f(W)] \sqrt{-v^{\prime}(\theta)}$ must be included in the income. Before proceeding with the general case let us first consider a situation where in addition to being coherent and law invariant, the risk measure $\varrho$ is also comonotone. In this case each security the principal sells to some agent increases her risk by the amount

$$
\varrho\left(-(f(W)-E[f(W)]) \sqrt{-v^{\prime}(\theta)}\right)+\left(v(\theta)-\theta v^{\prime}(\theta)\right) \geq 0 .
$$

This suggests that it is optimal for the principal not to sell a bond whose payoff moves into the same direction as her initial risk exposure. 
Proposition 4.1 Suppose that $\varrho$ is comonotone additive. If $f(W)$ and $W$ are comonotone, then $v=0$ is a solution to the principal's problem.

Proof. If $W$ and $f(W)$ are comonotone, then the risk measure in equation (4.1.1) is additive and the principal needs to solve

$$
\varrho(W)+\inf _{v \in \mathcal{C}} \int_{\Theta}\left(v(\theta)+\varrho(f(W)-\mathbb{E}[f(W)]) \sqrt{-v^{\prime}(\theta)}-\theta v^{\prime}(\theta)\right) d \theta .
$$

Since $\varrho(f(W)-E[f(W)]) \geq 0$ and $-\theta v^{\prime}(\theta) \geq 0$ we see that

$$
\int_{\Theta}\left(v(\theta)+\rho(F(W)) \sqrt{-v^{\prime}(\theta)}-\theta v^{\prime}(\theta)\right) d \theta \geq 0
$$

and hence $v \equiv 0$ is a minimizer.

In view of the preceding proposition the principal needs to design the payoff function $f$ in such a way that $W$ and $f(W)$ are not comonotone. We construct an optimal payoff function in the following subsection.

\subsubsection{A solution to the principal's problem}

Considering the fact that $\varrho(\cdot)$ is a decreasing function the principal's goal must be to make the quantity $C(v)$ as small as possible while keeping the income as large as possible. In a first step we therefore solve, for any constant $A \in \mathbb{R}$ the optimization problem

$$
\sup _{v \in \mathcal{C}} C(v) \quad \text { subject to } \int_{\Theta}\left(\mathbb{E}[f(W)] \sqrt{-v^{\prime}(\theta)}-v(\theta)+\theta v^{\prime}(\theta)\right) d \theta=A .
$$

The constraint variational problem (17) captures the problem of risk minimizing subject to an income constraint. It can be solved in closed form. The associated Euler-Lagrange equation is given by

$$
\lambda=\frac{d}{d \theta}\left(-\lambda \theta+\frac{\lambda \mathbb{E}[f]-1}{2 \sqrt{-v^{\prime}(\theta)}}\right),
$$

where $\lambda$ is the Lagrange multiplier. The income constraint and boundary conditions are:

$$
v^{\prime}(a)=-\frac{\left(\lambda^{\prime}\right)^{2}}{4 \lambda^{2} a^{2}} \quad \text { and } \quad v(1)=0 \quad \text { where } \quad \lambda^{\prime}=(\lambda \mathbb{E}[f]-1) .
$$

Integrating both sides of equation (18) and taking into account the normalization condition $v(1)=0$, we obtain

$$
v(\theta)=\frac{1}{8}\left(\frac{\lambda^{\prime}}{\lambda}\right)^{2}\left[\frac{1}{2 \theta-a}-\frac{1}{2-a}\right] .
$$

Inserting this equation into the constraint yields

$$
A=\mathbb{E}[f] \sqrt{\left(\frac{\lambda^{\prime}}{\lambda}\right)^{2}} \int_{a}^{1} \frac{d \theta}{2 \theta-a}-\left(\frac{\lambda^{\prime}}{\lambda}\right)^{2} \int_{a}^{1}\left\{\frac{1}{8}\left[\frac{1}{2 \theta-a}-\frac{1}{2-a}\right]+\frac{1}{4} \frac{\theta}{(2 \theta-a)^{2}}\right\} d \theta
$$


In terms of

$$
M:=\int_{a}^{1}\left\{\frac{1}{8}\left[\frac{1}{2 \theta-a}-\frac{1}{2-a}\right]+\frac{1}{4} \frac{\theta}{(2 \theta-a)^{2}}\right\} d \theta \quad \text { and } \quad N:=\int_{a}^{1} \frac{d \theta}{2 \theta-a}
$$

we have the quadratic equation

$$
-M\left(\frac{\lambda^{\prime}}{\lambda}\right)^{2}+N \mathbb{E}[f] \sqrt{\left(\frac{\lambda^{\prime}}{\lambda}\right)^{2}}-A=0
$$

which has the solution

$$
\sqrt{\left(\frac{\lambda^{\prime}}{\lambda}\right)^{2}}=\frac{N \mathbb{E}[f]-\sqrt{(N \mathbb{E}[f])^{2}-4 A M}}{2 M}
$$

We have used the root with alternating signs, as we require the problem to reduce to $\varrho(W)$ for $A=0$.

Remark 4.2 We notice that the constraint variational problem (17) is independent of the risk measure employed by the principal. This is because we minimized the risk pointwise subject to a constraint on aggregate revenues.

In view of the preceding considerations the principal's problem reduces to a one-dimensional minimization problems over the Reals:

$$
\inf _{A} \varrho\left(W-f(W) \frac{N^{2} \mathbb{E}[f]}{2 M}+f(W) \frac{N}{2 M} \sqrt{(N \mathbb{E}[f])^{2}-4 A M}\right)-A .
$$

Once the optimal value $A^{*}$ has been determined, the principal offers the securities

$$
\left(\frac{\lambda \mathbb{E}[f]-1}{4 \theta \lambda-2 \lambda a}\right) f(W)
$$

at a price

$$
\frac{\lambda \mathbb{E}[f]-1}{2}\left(\frac{3 E \lambda(2 \theta-a)-a}{(4 \theta \lambda-2 \lambda a)^{2}}+\frac{\lambda E-1}{\lambda^{2}} \frac{1}{2-a}\right) .
$$

Example 4.3 Assume that the principal measures her risk exposure using Average Value at Risk at level 0.05 . Let $\tilde{W}$ be a normally distributed random variable with mean $1 / 2$ and variance $1 / 20$. One can think that $\tilde{W}$ represents temperature. Suppose that the principal's initial income is exposed to temperature risk and it is given by $W=0.1(\tilde{W}-1.1)$ with associated risk

$$
\varrho(W)=0.0612
$$

Suppose furthermore that the principal sells units of a put option on $\tilde{W}$ with strike 0.5 , i.e.,

$$
f(W)=(W-0.5)^{+}
$$


By proceeding as above we approximated the principal's risk as -0.6731 and she offers the security

$$
X(\theta)=\frac{0.5459}{2 \theta-a} f(W)
$$

to the agent of type $\theta$ for a price

$$
\pi(\theta)=\frac{1.1921}{8(2-a)}-\frac{(1.1921) \theta-(0.22)(2 \theta-a)}{\sqrt{2}(2 \theta-a)^{2}} .
$$

\subsection{Put options with type dependent strikes}

In this section we consider the case where the principal underwrites put options on her income with type-dependent strikes. We assume that $W \leq 0$ is a bounded random variable and consider contracts of the form

$$
X(\theta)=(K(\theta)-|W|)^{+} \quad \text { with } \quad 0 \leq K(\theta) \leq\|W\|_{\infty} .
$$

The boundedness assumption on the strikes is made with no loss of generality and each equilibrium pricing scheme is necessarily non-negative. Note that in this case the risk measure can be defined on $\mathcal{L}(\mathbb{P})$, so we only require convergence in probability to use the Fatou property. We deduce that both the agents' net utilities and the variance of their positions is bounded from above by some constants $K_{1}$ and $K_{2}$, respectively. Thus, the principal chooses a function $v$ and contract $X$ from the set

$$
\left\{(X, v)\left|v \in \mathcal{C}, v \leq K_{1},-\operatorname{Var}[K(\theta)-|W|]=v^{\prime}(\theta),\right| v^{\prime} \mid \leq K_{2}, 0 \leq K(\theta) \leq\|W\|_{\infty}\right\} .
$$

The variance constraint $v^{\prime}(\theta)=-\operatorname{Var}\left[(K(\theta)-W)^{+}\right]$allows us to express the strikes in terms of a continuous function of $v^{\prime}$, i.e.,

$$
K(\theta)=F\left(v^{\prime}(\theta)\right) .
$$

The Principal's problem can therefore be written as

$$
\inf \left\{\varrho\left(W-\int_{\Theta}\left\{\left(F\left(v^{\prime}(\theta)\right)-|W|\right)^{+}-\mathbb{E}\left[\left(F\left(v^{\prime}(\theta)\right)-|W|\right)^{+}\right]\right\} d\right)-I(v)\right\}
$$

where the infimum is taken over the set of all functions $v \in \mathcal{C}$ that satisfy $v \leq K_{1}$ and $\left|v^{\prime}\right| \leq K_{2}$.

Remark 4.4 Within our current framework the contracts are expressed in terms of the derivative of the principal's choice of $v$. This reflects the fact that the principal restricts itself to typedependent put options and is not always true in the general case. 


\subsubsection{An existence result}

Let $\left\{v_{n}\right\}$ be a minimizing sequence for the principal's optimization problem. The functions $v_{n}$ are uniformly bounded and uniformly equicontinuous so we may with no loss of generality assume that $v_{n} \rightarrow \bar{v}$ uniformly. Recall this also implies a.s. convergence of the derivatives. By dominated convergence and the continuity of $F$, along with the fact that $W$ is bounded yields

$$
\int_{\Theta}\left(F\left(v_{n}^{\prime}(\theta)\right)-|W|\right)^{+} d \longrightarrow \int_{\Theta}\left(F\left(\bar{v}^{\prime}(\theta)\right)-|W|\right)^{+} d \quad \mathbb{P} \text {-a.s. }
$$

and

$$
\lim _{n \rightarrow \infty} \int_{\Theta} \mathbb{E}\left[\left(F\left(v_{n}^{\prime}(\theta)\right)-|W|\right)^{+} d \theta=\int_{\Theta} \mathbb{E}\left[\left(F\left(\bar{v}^{\prime}(\theta)\right)-|W|\right)^{+} d \theta\right.\right.
$$

This shows that the principal's positions converge almost surely and hence in probability. Since $\varrho$ is lower-semi-continuous with respect to convergence in probability we deduce that $\bar{v}$ solves the principal's problem.

\subsubsection{An algorithm for approximating the optimal solution}

We close this paper with a numerical approximation scheme for the principal's optimal solution within the pit option framework. We assume the set of states of the World is finite with cardinality $m$. Each possible state $\omega_{j}$ can occurs with probability $p_{j}$. The realizations of the principal's wealth are denoted by $W=\left(W_{1}, \ldots, W_{m}\right)$. Note that $p$ and $W$ are treated as known data. We implement a numerical algorithm to approximate a solution to the principal's problem when she evaluates risk via the risk measure

$$
\varrho(X)=-\sup _{q \in Q_{\lambda}} \sum_{j=1}^{m} X\left(\omega_{j}\right) p_{j} q_{j}
$$

where

$$
Q_{\lambda}:=\left\{q \in \mathbb{R}_{+}^{m} \mid p \cdot q=1, q_{j} \leq \lambda^{-1}\right\} .
$$

We also assume the set of agent types is finite with cardinality $n$, i.e. $\theta=\left(\theta_{1}, \ldots, \theta_{n}\right)$. The density of the types is given by $M:=\left(M_{1}, \ldots, M_{n}\right)$. In order to avoid singular points in the principal's objective function, we approximate the option's payoff function $f(x)=(K-x)^{+}$by the differentiable function

$$
T(x, K)= \begin{cases}0, & \text { if } \quad x \leq K-\epsilon, \\ S(x, K), & \text { if } \quad K-\epsilon<x<K+\epsilon, \\ x-K, & \text { if } \quad x \geq K+\epsilon .\end{cases}
$$

where

$$
S(x, K)=\frac{x^{2}}{4 \epsilon}+\frac{\epsilon-K}{2 \epsilon} x+\frac{K^{2}-2 A \epsilon+\epsilon^{2}}{4 \epsilon} .
$$


The algorithm uses a penalized Quasi-Newton method, based on Zakovic and Pantelides [20], to approximate a minimax point of

$$
\begin{aligned}
F(v, K, q)= & -\sum_{i=1}^{n} W_{i} p_{i} q_{i}+\frac{1}{n} \sum_{i=1}^{n}\left(\sum_{j=1}^{n} T\left(K_{j}-\left|W_{i}\right|\right)\right) p_{i} q_{i}-\frac{1}{n} \sum_{i=1}^{n}\left(\sum_{j=1}^{n-1} T\left(K_{j}-\left|W_{i}\right|\right)\right) p_{i} \\
& +\frac{1}{n} \sum_{i=1}^{n}\left(v_{i}-\theta_{i} \frac{v_{i+1}-v_{i}}{\theta_{i+1}-\theta_{i}}\right)+\frac{1}{n}\left(v_{n}-\frac{v_{n}-v_{n-1}}{1-\theta_{n-1}}\right)
\end{aligned}
$$

where $v=\left(v_{1}, \ldots, v_{n}\right)$ stands for the values of a convex, non-increasing function, $K=\left(K_{1}, \ldots, K_{n}\right)$ denotes the vector of type dependent strikes and the derivatives $v^{\prime}\left(\theta_{i}\right)$ are approximated by

$$
v^{\prime}\left(\theta_{i}\right)=\frac{v_{i+1}-v_{i}}{\theta_{i+1}-\theta_{i}}
$$

The need for a penalty method arises from the fact that we face the equality constraints $v^{\prime}(\theta)=-\operatorname{Var}\left[(K(\theta)-|W|)^{+}\right]$and $p \cdot q=1$. In order to implement a descent method, these constraints are relaxed and a penalty term is added. We denote by $n g$ the total number of constraints. The principal's problem is to find

$$
\min _{(v, K)} \max _{q \in Q_{\lambda}} F(v, K, q) \quad \text { subject to } \quad G(v, K, q) \leq 0
$$

where $G: \mathbb{R}^{2 n+m} \rightarrow \mathbb{R}^{n g}$ determines the constraints that keep $(v, K)$ within the set of feasible contracts and $q \in Q_{\lambda}$. The Maple code for our procedure is given in the appendix for completeness.

Example 4.5 Let us illustrate the effects of risk transfer on the principal's position in two model with five agent types and two states of the world. In both cases $W=(-1,-2), \theta=$ $(1 / 2,5 / 8,3 / 4,7 / 8,1)$ and $\lambda=1.1$ The starting values $v_{0}, q_{0}$ and $K_{0}$ we set are $(4,3,2,1,0),(1,1)$ and $(1,1,1,1,1)$ respectively.

i) Let $p=(0.5,0.5)$ and the types be uniformly distributed. The principal's initial evaluation of her risk is 1.52. The optimal function $v$ and strikes are:

\begin{tabular}{|l|l|}
\hline$V_{1}$ & 0.1055 \\
\hline$V_{2}$ & 0.0761 \\
\hline$V_{3}$ & 0.0501 \\
\hline$V_{4}$ & 0.0342 \\
\hline$V_{5}$ & 0.0195 \\
\hline
\end{tabular}

\begin{tabular}{|l|l|}
\hline$K_{1}$ & 1.44 \\
\hline$K_{2}$ & 1.37 \\
\hline$K_{3}$ & 1.07 \\
\hline$K_{4}$ & 1.05 \\
\hline$K_{5}$ & 1.05 \\
\hline
\end{tabular}

The Principal's valuation of her risk after the exchanges with the agents decreases to 0.2279 .

ii) In this instance $p=(0.25,0.75)$ and $M=(1 / 15,2 / 15,3 / 15,4 / 15,5 / 15)$. The principal's initial evaluation of her risk is 1.825 . The values for the discretized $v$ the type-dependent strikes are: 


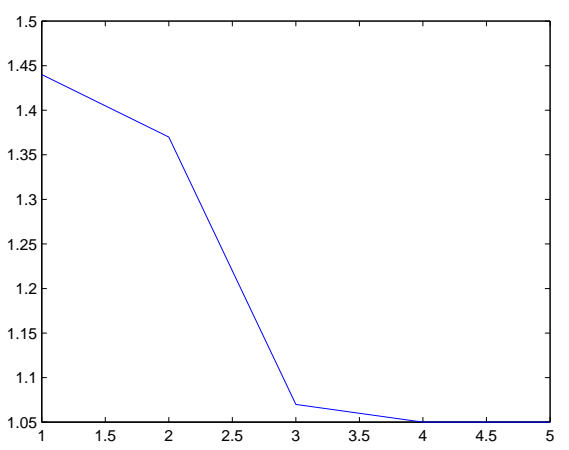

(a) The type-dependent strikes.

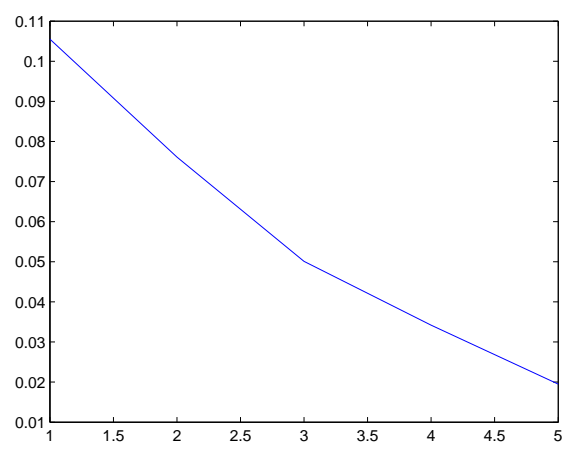

(b) The optimal function $v$.

Figure 1: Optimal solution for underwriting put options, Case 1.

\begin{tabular}{|l|l|}
\hline$V_{1}$ & 0.0073 \\
\hline$V_{2}$ & 0.0045 \\
\hline$V_{3}$ & 0.0029 \\
\hline$V_{4}$ & 0.0026 \\
\hline$V_{5}$ & 0.0025 \\
\hline
\end{tabular}

\begin{tabular}{|l|l|}
\hline$K_{1}$ & 1.27 \\
\hline$K_{2}$ & 1.16 \\
\hline$K_{3}$ & 1.34 \\
\hline$K_{4}$ & 0.11 \\
\hline$K_{5}$ & 0.12 \\
\hline
\end{tabular}

The Principal's valuation of her risk after the exchanges with the agents is 0.0922 .

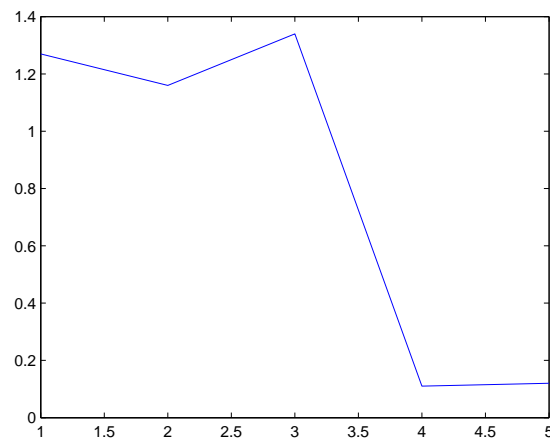

(a) The type-dependent strikes.

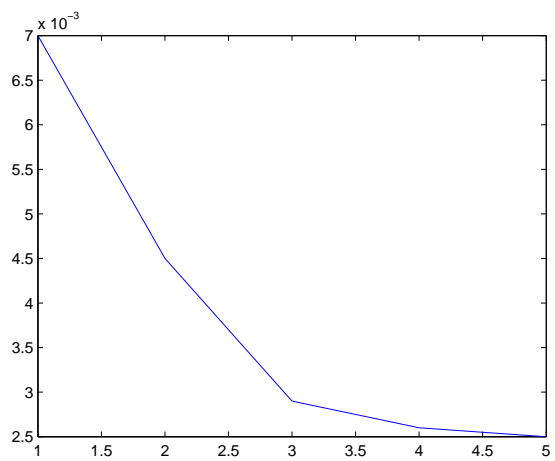

(b) The optimal function $v$.

Figure 2: Optimal solution for underwriting put options, Case 2. 


\section{Conclusions}

In this paper we analyzed a screening problem where the principal's choice space is infinite dimensional. Our motivation was to present a nonlinear pricing scheme for over-the-counter financial products, which she trades with a set of heterogeneous agents with the aim of minimizing the exposure of her income to some non-hedgeable risk. In order to characterize incentive compatible and individually rational catalogues, we have made use of U-convex analysis. To keep the problem tracktable we have assumed the agents have mean-variance utilities, but this is not necessary for the characterization of the problem. Considering more general utility functions is an obvious extension to this work. Our main result is a proof of existence of a solution to the principal's risk minimization problem in a general setting. The examples we have studied suggest that the methodologies for approaching particular cases are highly dependent on the choice of risk measure, as well as on the kinds of contracts the principal is willing (or able) to offer. In most cases obtaining closed form solutions is not possible and implementations must be done using numerical methods. As a work in progress we are considering agents with heterogenous initial endowments (or risk exposures), as well as a model that contemplates an economy with multiple principals.

\section{A Coherent risk measures on $L^{2}$.}

In this appendix we recall some properties and representation results for risk measures on $L^{2}$ spaces; we refer to the textbook of Föllmer and Schied [12] for a detailed discussion of convex risk measures on $L^{\infty}$ and to Cheridito and Tianbui [8] for risk measures on rather general state spaces. Bäuerle and Müller [4] establish representation properties of risk law invariant risk measures on $L^{p}$ spaces for $p \geq 1$. We assume that all random variables are defined on some standard non-atomic probability space $(\Omega, \mathcal{F}, \mathbb{P})$.

Definition A.1 (i) A monetary measure of risk on $L^{2}$ is a function $\varrho: L^{2} \rightarrow \mathbb{R} \cup\{\infty\}$ such that for all $X, Y \in L^{2}$ the following conditions are satisfied:

- Monotonicity: if $X \leq Y$ then $\varrho(X) \geq \varrho(Y)$.

- Cash Invariance: if $m \in \mathbb{R}$ then $\varrho(X+m)=\varrho(X)-m$.

(ii) A risk measure is called coherent if it is convex and homogeneous of degree 1, i.e., if the following two conditions hold:

- Convexity: for all $\lambda \in[0,1]$ and all positions $X, Y \in L^{2}$ :

$$
\varrho(\lambda X+(1-\lambda) Y) \leq \lambda \varrho(X)+(1-\lambda) \varrho(Y)
$$


- Positive Homogeneity: For all $\lambda \geq 1$

$$
\varrho(\lambda X)=\lambda \varrho(X)
$$

(iii) The risk measure is called coherent and law invariant, if, in addition,

$$
\rho(X)=\rho(Y)
$$

for any two random variables $X$ and $Y$ which have the same law.

(iv) The risk measure $\varrho$ on $L^{2}$ has the Fatou property if for any sequence of random variables $X_{1}, X_{2}, \ldots$ that converges in $L^{2}$ to a random variable $X$ we have

$$
\rho(X) \leq \liminf _{n \rightarrow \infty} \rho\left(X_{n}\right)
$$

Given $\lambda \in(0,1]$, the Average Value at Risk of level $\lambda$ of a position $Y$ is defined as

$$
A V @ R_{\lambda}(Y):=-\frac{1}{\lambda} \int_{0}^{\lambda} q_{Y}(t) d t,
$$

where $q_{Y}(t)$ is the upper quantile function of $Y$. If $Y \in L^{\infty}$, then we have the following characterization

$$
A V @ R_{\lambda}(Y)=\sup _{Q \in \mathcal{Q}_{\lambda}}-\mathbb{E}_{Q}[Y]
$$

where

$$
\mathcal{Q}_{\lambda}=\left\{Q<<P \mid \frac{d Q}{d P} \leq \frac{1}{\lambda}\right\}
$$

Proposition A.2 For a given financial position $Y \in L^{2}$ the mapping $\lambda \mapsto A V @ R_{\lambda}(Y)$ is decreasing in $\lambda$.

It turns out the Average Value of Risk can be viewed as a basis for the space of all lawinvariant, coherent risk measures with the Fatou property. More precisely, we have the following result.

Theorem A.3 The risk measure $\varrho: L^{2} \rightarrow \mathbb{R}$ is law-invariant, coherent and has the Fatou Property if and only if $\varrho$ admits a representation of the following form:

$$
\varrho(Y)=\sup _{\mu \in M}\left\{\int_{0}^{1} A V @ R_{\lambda}(Y) \mu(d \lambda)\right\}
$$

where $M$ is a set of probability measures on the unit interval.

As a consequence of Proposition A.2 and Theorem A.3 we have the following Corollary: 
Corollary A.4 If $\varrho: L^{2} \rightarrow \mathbb{R}$ is a law-invariant, coherent risk measure with the Fatou Property then

$$
\varrho(Y) \geq-\mathbb{E}[Y]
$$

An important class of risk measures are comonotone risk measures risk. Comonotone risk measures are characterized by the fact that the risk associated with two position whose payoff "moves in the same direction" is additive.

Definition A.5 A risk measure $\varrho$ is said to be comonotone if

$$
\varrho(X+Y)=\rho(X)+\rho(Y)
$$

whenever $X$ and $Y$ are comonotone, i.e., whenever

$$
\left(X(\omega)-X\left(\omega^{\prime}\right)\right)\left(Y(\omega)-Y\left(\omega^{\prime}\right)\right) \geq 0 \quad \mathbb{P} \text {-a.s. }
$$

Comonotone, law invariant and coherent risk measures with the Fatou property admit a representation of the form

$$
\varrho(Y)=\int_{0}^{1} A V @ R_{\lambda}(Y) \mu(d \lambda)
$$

\section{B Maple code for the example of Section 4.2 .2}

with(LinearAlgebra)

$\mathrm{n}:=5:$

$\mathrm{m}:=2$ :

$\mathrm{ng}:=2^{*} \mathrm{~m}+4^{*} \mathrm{n}+1$ :

This section constructs the objective function $f$ and its gradient.

$\mathrm{x}:=\operatorname{Vector}\left(2^{*} \mathrm{n}\right.$, symbol $\left.=\mathrm{xs}\right)$ :

$\mathrm{q}:=\operatorname{Vector}(\mathrm{m}, \mathrm{symbol}=\mathrm{qs})$ :

$$
\begin{aligned}
f:= & \operatorname{add}(-G[j] * p[j] * q[j], j=1 . . m)+ \\
& \operatorname{add}(\operatorname{add}(T(x[i+n], W[j]) * M[i] * p[j] * q[j], i=1 . . n), j=1 . . m)- \\
& \operatorname{add}(\operatorname{add}(T(x[i+n], W[j]) * M[i] * p[j], i=1 . . n), j=1 . . m)+ \\
& a d d((x[i]-t[i] *(x[i+1]-x[i]) /(t[i+1]-t[i])) * M[i], i=1 . . n-1)+ \\
& x[n]-t[n] *(x[n]-x[n-1]) * M[n] /(t[n]-t[n-1]):
\end{aligned}
$$

$T T:=(x, K)->1 / 4 * x^{2} /$ eps $+1 / 2 *($ eps $-K) * x /$ eps $+1 / 4 *\left(K^{2}-2 * K *\right.$ eps + eps $\left.^{2}\right) /$ eps $:$

$T:=(x, K)->$ piecewise $(x \leq K-$ eps $, 0, x<K+$ eps, $T T(x, K), K+$ eps $\leq x, x-K)$ :

$\operatorname{gradfx}:=0$ :

$\operatorname{gradfq}:=0$ : 
for i from 1 to $2^{*} \mathrm{n}$

do $\operatorname{gradfx}[\mathrm{i}]:=\operatorname{diff}(\mathrm{f}, \mathrm{x}[\mathrm{i}])$

end do:

for $\mathrm{i}$ from 1 to $\mathrm{m}$

do $\operatorname{gradf} q[\mathrm{i}]:=\operatorname{diff}(\mathrm{f}, \mathrm{q}[\mathrm{i}])$

end do:

This section constructs the constraint function $g$ and its gradient.

$\mathrm{g}:=\operatorname{Vector}(\mathrm{ng}$, symbol $=\mathrm{tt}):$

for $\mathrm{j}$ from 1 to $\mathrm{n}$

$\operatorname{dog} g[\mathrm{j}]:=-\mathrm{x}[\mathrm{j}]$ end do:

for $\mathrm{j}$ from 1 to $\mathrm{n}-1$

do $g[\mathrm{j}+\mathrm{n}]:=\mathrm{x}[\mathrm{j}+1]-\mathrm{x}[\mathrm{j}]$

end do:

for i from 1 to $\mathrm{n}-1$

$\operatorname{do} \mathrm{g}[\mathrm{i}+2 * \mathrm{n}-1]:=\operatorname{add}\left(\mathrm{T}(\mathrm{x}[\mathrm{i}+\mathrm{n}], W[j])^{2} * p[j], j=1 . . m\right)-\operatorname{add}(T(x[i+n], W[j]) * p[j], j=1 . . m)^{2}+(x[i+$ $1]-x[i]) /(t[i+1]-t[i])-e p s 2$

end do:

$g[3 * n-1]:=\operatorname{add}\left(T(x[2 * n], W[j])^{2} * p[j], j=1 . . m\right)-\operatorname{add}(T(x[2 * n], W[j]) * p[j], j=1 . . m)^{2}+(x[n]-$ $x[n-1]) /(t[n]-t[n-1])-e p s 2:$

for i from 1 to $\mathrm{n}-1$

do $g[i+3 * n-1]:=-a d d\left(T(x[i+n], W[j])^{2} * p[j], j=1 . . m\right)+\operatorname{add}(T(x[i+n], W[j]) * p[j], j=1 . . m)^{2}-$ $(x[i+1]-x[i]) /(t[i+1]-t[i])-e p s 2$

end do:

$g[4 * n-1]:=-\operatorname{add}\left(T(x[2 * n], W[j])^{2} * p[j], j=1 . . m\right)+\operatorname{add}(T(x[2 * n], W[j]) * p[j], j=1 . . m)^{2}-(x[n]-$ $x[n-1]) /(t[n]-t[n-1])-e p s 2:$

$\mathrm{g}\left[4^{*} \mathrm{n}\right]:=\operatorname{add}\left(\mathrm{p}[\mathrm{i}]^{*} \mathrm{q}[\mathrm{i}], \mathrm{i}=1 . . \mathrm{m}\right)-1+\operatorname{eps} 3:$

$\mathrm{g}\left[4^{*} \mathrm{n}+1\right]:=-\operatorname{add}\left(\mathrm{p}[\mathrm{i}]^{*} \mathrm{q}[\mathrm{i}], \mathrm{i}=1 . . \mathrm{m}\right)-1-\mathrm{eps} 3:$

for i from 1 to $\mathrm{m}$

do $\mathrm{g}\left[\mathrm{i}+4^{*} \mathrm{n}+1\right]:=-\mathrm{q}[\mathrm{i}]$

end do:

for i to $\mathrm{m}$

do $\mathrm{g}\left[\mathrm{i}+\mathrm{m}+4^{*} \mathrm{n}+1\right]:=\mathrm{q}[\mathrm{i}]-$ lambda

end do: $\operatorname{gradgx}:=0$ :

$\operatorname{gradgq}:=0$ :

for i from 1 to $\mathrm{ng}$

do for $\mathrm{j}$ from 1 to $2^{*} \mathrm{n}$

do $\operatorname{gradgx}[i, j]:=\operatorname{diff}(g[i], x[j])$

end do:

end do: 
for i from 1 to ng

do for $\mathrm{j}$ from 1 to $\mathrm{m}$

do $\operatorname{gradgq}[i, \mathrm{j}]:=\operatorname{diff}(\mathrm{g}[\mathrm{i}], \mathrm{q}[\mathrm{j}])$

end do:

end do:

This section constructs the slackness structures.

$\mathrm{e}:=\operatorname{Vector}(\mathrm{ng}, 1)$ :

$\mathrm{s}:=\operatorname{Vector}(\mathrm{ng}, \mathrm{symbol}=\mathrm{si})$ :

$\mathrm{z}:=\operatorname{Vector}(\mathrm{ng}$, symbol $=\mathrm{zi})$ :

$\mathrm{S}:=\operatorname{DiagonalMatrix}(\mathrm{s})$ :

$\mathrm{Z}:=$ DiagonalMatrix $(\mathrm{z})$ :

This section initializes the variable and parameter vectors.

$\mathrm{x}:=\operatorname{Vector}\left(2^{*} \mathrm{n}\right.$, symbol $\left.=\mathrm{xs}\right)$ :

$\mathrm{q}:=\operatorname{Vector}(\mathrm{m}$, symbol $=\mathrm{qs})$ :

$\mathrm{p}:=\operatorname{Vector}(\mathrm{m}, \operatorname{symbol}=\mathrm{ps})$ :

$\mathrm{W}:=\operatorname{Vector}(\mathrm{m}, \mathrm{symbol}=\mathrm{ws})$ :

$\mathrm{G}:=\operatorname{Vector}(\mathrm{m}, \mathrm{symbol}=\mathrm{gs})$ :

$\mathrm{t}:=\operatorname{Vector}(\mathrm{n}$, symbol $=\mathrm{ts})$ :

$\mathrm{M}:=\operatorname{Vector}(\mathrm{n}$, symbol $=\mathrm{ms})$ :

$\operatorname{chi}:=\operatorname{convert}([\mathrm{x}, \mathrm{q}, \mathrm{s}, \mathrm{z}]$, Vector $)$ :

This section constructs the Lagrangian and its Hessian matrix.

$\mathrm{F}:=\operatorname{convert}([\operatorname{gradf} \mathrm{x}+(\operatorname{VectorCalculus}[$ DotProduct $])($ Transpose $(\operatorname{gradgx}), \mathrm{z})$, gradfq-(VectorCalculus[DotProduct])(Transpose(gradgq), z),

(VectorCalculus[DotProduct])((VectorCalculus[DotProduct])(Z, S), e)-mu*e, g+s], Vector):

$\mathrm{DF}:=0$ :

for i from 1 to $2^{*} \mathrm{n}+\mathrm{m}+2^{*} \mathrm{ng}$

do for $\mathrm{j}$ from 1 to $2^{*} \mathrm{n}+\mathrm{m}+2^{*} \mathrm{ng}$

do $\operatorname{DF}[\mathrm{i}, \mathrm{j}]:=\operatorname{diff}(\mathrm{F}[\mathrm{i}], \operatorname{chi}[\mathrm{j}])$

end do:

end do:

This section inputs the initial values of the variables and the values of the parameters.

xinit := $(4,3,2,1,0,1,1,1,1,1)$ :

qinit $:=(1,1)$ : 


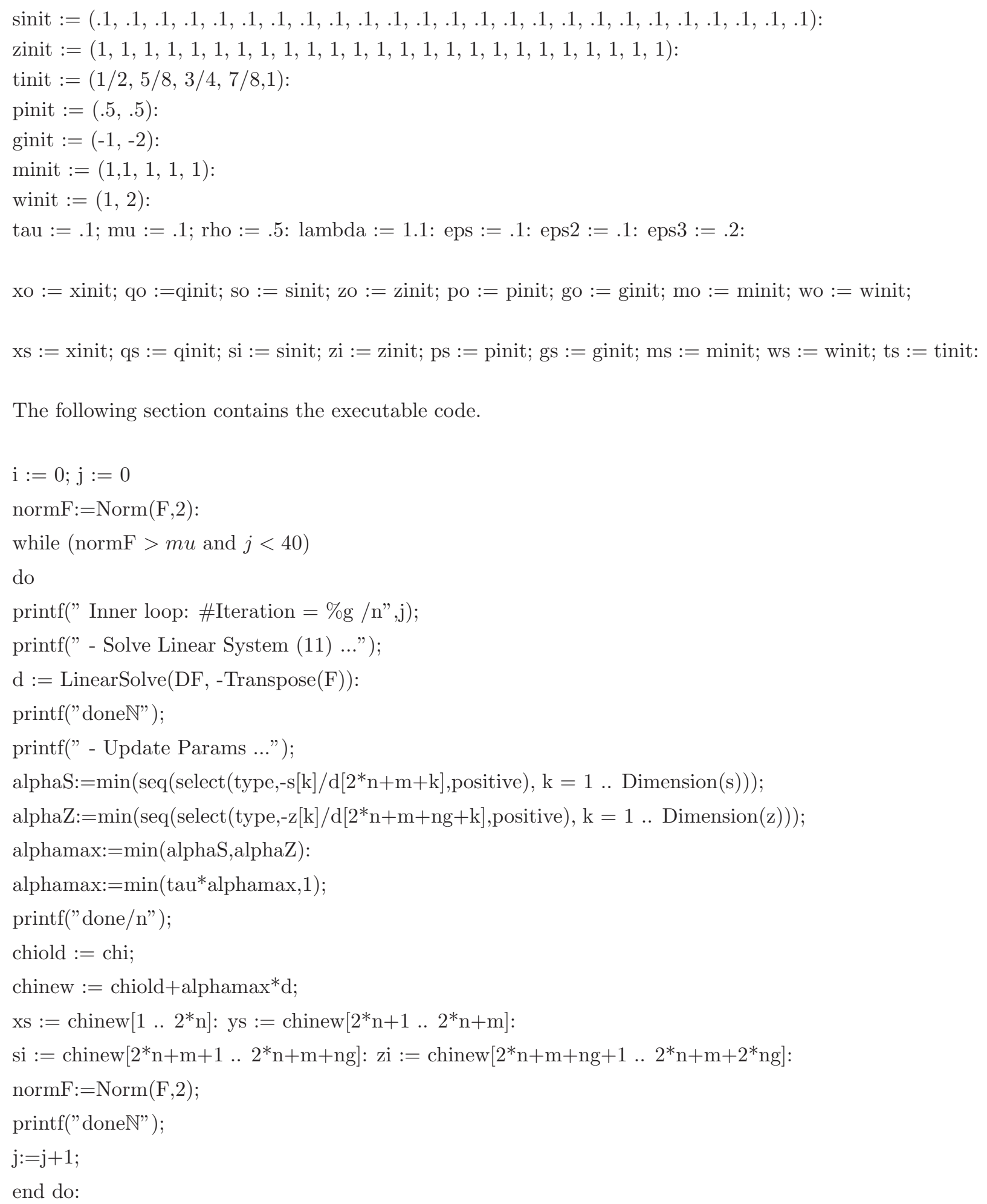




\section{References}

[1] Armstrong, M.: Multiproduct Nonlinear Pricing, Econometrica, 64, 51-75, 1996.

[2] Barrieu, P. \& N. El Karoui: Optimal Design of Derivatives in Illiquid Framework, Quantitative Finance, 2, 1-8, 2005.

[3] Barrieu, P. \& N. El Karoui: Inf-convolution of risk measures and optimal risk transfer, Finance and Stochastics, 9, 269-298, 2005.

[4] Bäuerle, N. \& A. Müller: Stochastic Orders and Risk Measures: Consistency and Bounds, Insurance: Mathematics \& Economics,38, 132-148, 2006.

[5] Becherer, D.: Utility indifference Hedging and Valuation via Reaction Diffusion Systems, Proceedings of the Royal Society, Series A, 460, 27-51, 2004.

[6] Becherer, D.: Bounded Solutions to Backward SDE's with Jumps for Utility Optimization and Indifference Hedging, Annals of Applied Probability, to appear.

[7] Carlier, G., Ekeland, I \& N. Touzi: Optimal Derivatives Design for Mean-Variance Agents under Adverse Selection, Preprint, 2006.

[8] Cheridito, P. \& L. Tianhui: Monetary Risk Measures on Maximal Subspaces Of Orlicz Classes, Preprint, 2006.

[9] Davis, M.: Pricing Weather Derivatives by Marginal Value, Quantitative Finance, 1, 305-308, 2001.

[10] Ekeland, I., Témam, R., Convex Analysis and Variational Problems, Classics in Applied Mathematics, 28, SIAM, 1976.

[11] Filipović, D. \& M. Kupper: Equilibrium and Optimality for Monetary Utility Functions under Constraints, Preprint, 2006.

[12] Föllmer, H. \& A. Schied: Stochastic Finance. An Introduction in Discrete Time, de Gruyter Studies in Mathematics, 27, 2004.

[13] Guesnerie, R.: A contribution to the Pure Theory of Taxation, Econometrica, 49, 33-64, 1995.

[14] Horst U. \& M. Müller: On the Spanning Property of Risk Bonds Priced by Equilibrium, Mathematics of Operations Research, to appear.

[15] Horst U., Pirvu, T. \& G. Nunes dos Reis: On Securitization, Market Completion and Equilibrium Risk Transfer, Working Paper, 2007. 
[16] Hu, Y., Imkeller, P. \& M. Müller: Market Completion and Partial Equilibrium, International Journal of Theoretical \& Applied Finance, to appear.

[17] Jouini, E., Schachermeyer, W. \& N. Touzi: Law Invariant Risk Measures have the Fatou Property, Advances in Mathematical Economics 9, 49-71, 2006.

[18] Mussa M. \& S. Rosen: Monopoly and Product Quality, Journal of Economic Theory, 18, 301-317, 1978.

[19] Rochet, J.-C. \& P. Choné: Iroining, Sweeping and Multidimensional Screening, Econometrica,66, 783-826, 1988.

[20] Zakovic, S. \& C. Pantelides: An Interior Point Algorithm for Computing Saddle Points of Constrainde Continuous Minimax, Annals of Operations Research, 99, 59-77, 2000. 